\title{
Archéologie des résidences aristocratiques médiévales en Poitou-Charentes (1987-2004)
}

\section{Luc Bourgeois}

\section{Citer ce document / Cite this document :}

Bourgeois Luc. Archéologie des résidences aristocratiques médiévales en Poitou-Charentes (1987-2004) . In: Archéologie du Midi médiéval $n^{\circ} 4,2006$. Résidences aristocratiques, résidences du pouvoir entre Loire et Pyrénées, XeXVe siècles. Recherches archéologiques récentes, 1987-2002. pp. 53-62;

doi : https://doi.org/10.3406/amime.2006.1574

https://www.persee.fr/doc/amime_1278-3358_2006_sup_4_1_1574

Fichier pdf généré le 22/03/2019 
Luc Bourgeois Centre d'études supérieures de civilisation médiévale (UMR 6589 du CNRS), Poitiers.

\section{Archéologie des résidences aristocratiques médiévales en Poitou-Charentes (1987-2004)}

\section{Essai de bilan}

Les états des lieux réalisés en 1987 et 1990 par André Debord ${ }^{1}$ constituent une base pratique pour considérer l'évolution des recherches depuis la fin des années quatre-vingt et nous nous réfèrerons souvent à ces bilans.

\section{Evolution des cadres d'intervention}

Les 114 autorisations administratives délivrées entre 1987 et septembre 2004 concernent 44 sites, assez inégalement répartis dans l'espace régional. Un comptage basé sur les autorisations privilégie le Haut-Poitou (tableau I). En nombre de sites ayant fait l'objet d'interventions, c'est le département de la Charente-Maritime qui l'emporte et la Charente voisine qui fait pâle figure (fig. 1).

Le bilan dressé en 1987 enregistrait l'interruption récente d'un certain nombre d'opérations menées par des associations locales. Malgré un léger reflux du nombre d'autorisations accordées, les chercheurs bénévoles demeurent pourtant fortement présents dans ce secteur de la recherche, avec toutefois une récente régression qui reste à confirmer (tableau II). En contrepoint, les années 1990-1991 - marquées par une croissance sans lendemain des opérations - ont vu se développer l'archéologie contractuelle (AFAN puis INRAP,
Association des archéologues de Poitou-Charentes) et les opérations intégrées aux études et aux travaux de la Conservation régionale des Monuments historiques. Ces chantiers menés en amont des restaurations n'ont jamais suscité d'opérations de grande ampleur et leurs problématiques se limitent souvent à des questions techniques (niveaux de sol, complément de plan de structures en partie disparues). Ils ne concernent pratiquement jamais les édifices inscrits à l'inventaire supplémentaire des Monuments historiques, la plupart du temps restaurés sans intervention archéologique. La dernière décennie a également vu croître les interventions archéologiques réalisées à la demande de collectivités territoriales propriétaires de monuments.

La part des recherches programmées a tendance à progresser légèrement depuis 2002, après une absence totale d'autorisations de ce type entre 1994 et 2001. D'autres entreprises à long terme se cachent pourtant derrière des statuts différents (sondage, sauvetage urgent, etc.) : la pratique du « sondage à répétition » dénoncée en 1987 se perpétue donc sous d'autres formes (Gençay, Oiron, Parthenay). Cette multiplication des opérations ponctuelles, parfois menées par

1. Debord 1990 et 1991.

2. Gil 2000 et article du même chercheur dans les actes de ce colloque. 
Tableau I : activité archéologique par année et département (en nombre d'autorisations)

\begin{tabular}{|c|c|c|c|c|c|c|c|c|c|c|c|c|c|c|c|c|c|c|c|c|}
\hline & $\mathscr{g}$ & $\dddot{\wp}$ & $\stackrel{\circ}{\stackrel{一}{\sigma}}$ & ఏ & $\bar{\sigma}$ & Бे & ळ & g & $\approx$ & 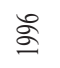 & s & $\stackrel{\infty}{a}$ & よे & 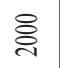 & Б્స & ఏ్రి & ڤ్ & 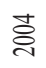 & 喼 & 总 \\
\hline Charente & 2 & 1 & 1 & 1 & 2 & 1 & 1 & 2 & 2 & - & 2 & 2 & 1 & - & - & 1 & 3 & 3 & 25 & 7 \\
\hline Charente-Maritime & - & 2 & 1 & - & 1 & 2 & - & 2 & 1 & 2 & - & 3 & 2 & - & 2 & 2 & 2 & 1 & 23 & 17 \\
\hline Deux-Sèvres & 2 & 1 & 1 & 4 & 7 & 1 & 1 & - & 2 & 3 & 2 & 3 & 2 & 3 & 3 & 4 & 1 & - & 40 & 9 \\
\hline Vienne & 1 & 1 & 1 & 2 & 4 & 1 & 2 & - & - & 4 & 3 & 3 & - & 1 & 1 & 1 & 1 & - & 26 & 11 \\
\hline TOTAL & 5 & 5 & 4 & 7 & 14 & 5 & 4 & 4 & 5 & 9 & 7 & 11 & 5 & 4 & 6 & 8 & 7 & 4 & 114 & 44 \\
\hline
\end{tabular}

Tableau II : statut des titulaires d'autorisations

\begin{tabular}{|c|c|c|c|c|c|c|c|c|c|c|c|c|c|c|c|c|c|c|c|}
\hline & $\stackrel{\circ}{\circ}$ & $\stackrel{\infty}{\stackrel{\leftrightarrow}{\sigma}}$ & 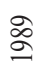 & \& & $\bar{\sigma}$ & ऽ & & g & ळे & $\stackrel{\circ}{2}$ & s & $\stackrel{\infty}{\sigma}$ & बे & ڤ્సి & ळ్ & ઠ్రి & శ్రి & ఫ્ત & 喼 \\
\hline Bénévoles & 4 & 4 & 1 & 4 & 2 & 3 & 1 & - & - & 3 & 1 & 3 & 1 & 2 & 2 & 1 & 1 & - & 36 \\
\hline Afan/Inrap & - & - & - & 1 & 2 & - & 1 & 3 & 3 & 3 & 2 & 2 & 3 & 1 & 3 & 2 & 1 & 1 & 27 \\
\hline Autres contractuels & - & - & - & 1 & 1 & - & 1 & - & - & - & 1 & 3 & 1 & - & - & 1 & 1 & 1 & 11 \\
\hline Culture & - & - & 1 & - & 2 & 2 & - & - & - & - & 2 & 1 & - & - & - & - & 2 & - & 10 \\
\hline Ens. Supérieur & 1 & 1 & 1 & 1 & 1 & 1 & 1 & 1 & 1 & 1 & - & - & - & - & - & 2 & 1 & 1 & 14 \\
\hline Collectivités & - & - & 1 & - & 1 & - & - & - & 1 & 2 & 1 & 2 & - & 1 & 1 & 1 & - & - & 11 \\
\hline Autre & - & - & - & - & - & - & - & - & - & - & - & - & - & - & - & 1 & 1 & 1 & 3 \\
\hline TOTAL & 5 & 5 & 4 & 7 & 14 & 5 & 4 & 4 & 5 & 9 & 7 & 11 & 5 & 4 & 6 & 8 & 7 & 4 & 114 \\
\hline
\end{tabular}

des intervenants différents, rend problématique toute synthèse des données. A Parthenay, cette parcellisation a été corrigée par la mise en place d'un projet collectif de recherches sur les fortifications de la ville.

Si l'on excepte une enquête menée en Châtelleraudais dans le cadre d'un travail universitaire ${ }^{2}$, aucune opération de prospection thématique liée aux résidences aristocratiques n'a été autorisée depuis quinze ans. L'essor des travaux d'inventaire associé à la préparation du colloque de 1987 a donc fait long feu, alors même qu'il était présenté comme une priorité de la recherche. En la matière, la compilation rapide de données anciennes telle qu'on continue à la pratiquer ne remplace en rien des inventaires critiques combinant les archives et le terrain. D'autre part, on reste étonné par la discrétion de l'archéologie des élévations dans les recherches récentes, mais cette technique demeure globalement sous-utilisée en Poitou-Charentes.

\section{Mutation ou stagnation des recherches?}

Le problème des origines du château, considéré comme fondamental par les programmations successives du CNRA, commence à peine à être abordé. En la matière, le site phare de la région demeure le castrum des comtes d'Angoulême à Andone³. La fouille préventive récemment menée sur une résidence aristocratique carolingienne aux portes de Châtellerault et l'occupation contemporaine de la grotte d'Agris illustrent la grande variété des situations avant l'an mil. Dans d'autres sites, la concentration des efforts sur les états tardifs des châteaux se prêtait mal à une enquête sur les premiers temps des résidences signalées dans les textes avant le milieu du XIe siècle (Gençay, Parthenay, Lusignan, etc.). Dans le cadre urbain, les bâtiments sur solins des IXe-XIe siècles découverts à Poitiers (Parking du Calvaire, hôtel Aubaret) pourraient rappeler les habitats privilégiés établis en coeur d'îlot connus dans d'autres régions. L'analyse morphogénétique a également suscité quelques hypothèses à vérifier sur l'emprise originelle des castra urbains ${ }^{4}$. Des études récentes menées ailleurs montrent combien l'organisation interne de ces castra précoces diffère des schémas convenus. Pourtant, malgré l'existence dans la région Poitou-Charentes d'exemples aussi remarquables que le complexe castral de Chauvigny, des phénomènes comme l'habitat des milites castri ou la pratique de la co-seigneurie ont été pour l'instant à peine abordés ${ }^{5}$.

On attendait un essor des recherches concernant les fortifications de terre : il n'est pas venu. Comme en 1987, le Haut-Poitou et l'Aunis demeurent vierges d'inventaires systématiques. La désaffection que connaît ce type d'enquête n'est pas limitée à la région Poitou-Charentes. La difficulté à opérer un classement typologique et chronologique de ces vestiges semble avoir fortement limité le nombre des opérations d'inventaire. Quelques opérations préventives ont toutefois révélé des structures originales comme la petite enceinte circulaire de Villiersen-Plaine et la motte ou maison-forte remarquablement conservée de Puyrolland.

La littérature récente continue à privilégier les structures maçonnées des XIIe-XVe siècles. Rarement produite par des archéologues auxquels des travaux trop ponctuels interdisent toute lecture synthétique, elles ne sont qu'exceptionnellement marquées par la collaboration qui s'impose entre historiens, archéologues et historiens de l'architecture. Si l'on excepte le cas de Parthenay, les plus amples monographies parues ces dernières années sont toutes issues de travaux universitaires publiés (le Coudray-Salbart, Gençay)

\section{Debord 1994 et 1995.}

4. Bourgeois 2000a et présentation dans le présent volume.

5. Rémy 2003 ; Bourgeois, sous presse. Ces thèmes commencent aussi à apparaître dans quelques travaux universitaires (par exemple Puissant 2004). 
ou non (Angles-sur-l'Anglin, Berrie, Château-Larcher, Dissay, le Haut-Clairvaux, Hérisson, Montreuil-Bonnin, Thouars, etc.). Ces travaux marquent un renouvellement des centres d'intérêt: les défenses avancées ou l'adaptation des structures à l'artillerie à poudre étaient par exemple des thèmes rarement développés dans les études antérieures.

S'inscrivant là encore dans une tendance nationale, la recherche sur les résidences aristocratiques de la fin du Moyen Age connait un essor notable. L'étude des premiers états de Chiré-en-Montreuil ou d'Oiron ${ }^{6}$, l'analyse des dispositifs d'entrée des petits châteaux ${ }^{7}$ ou un inventaire des maisons nobles de la vicomté de Châtellerault ${ }^{8}$ témoignent de cet intérêt nouveau, tout comme les travaux historiques traitant des autorisations de fortifier ${ }^{9}$ ou du financement des chantiers de reconstruction à la fin de la guerre de Cent Ans ${ }^{10}$.

L'environnement des résidences aristocratiques et leur place dans l'occupation du sol demeurent peu étudiés. La relation entre ville et château est toutefois abordée par quelques publications récentes ${ }^{11}$ et le thème du bourg castral cher à André Debord revient dans quelques recherches. Les résidences aristocratiques urbaines n'apparaissent qu'à travers un travail consacré au palais épiscopal d'Angoulême ${ }^{12}$, deux études en cours sur le palais ducal de Poitiers ${ }^{13}$ et la fouille d'une vaste salle à Niort. En milieu rural, la relation entre résidence aristocratique, monuments religieux et habitat ne semble pas avoir retenu l'attention et les travaux pionniers consacrés aux garennes seigneuriales du HautPoitou dans les années 1980 n'ont pas fait école ${ }^{14}$.

La rareté des publications concernant le mobilier quotidien des résidences aristocratiques relève également d'une tendance générale. La réalisation de travaux sur des ensembles monétaires ${ }^{15}$, du petit mobilier ${ }^{16}$, des terres cuites architecturales ${ }^{17}$ ou des collections de faune ${ }^{18}$ reste d'ailleurs limitée par la faible extension de la plupart des chantiers archéologiques. L'étude des procès-verbaux de visite et des inventaires après décès de la fin du Moyen Age ${ }^{19}$ livre parfois d'autres facettes de cette culture matérielle.

L'analyse des réseaux castraux a par contre engendré des réflexions nouvelles : les travaux réalisés sous l'égide des Plantagenêt ${ }^{20}$ ou de Philippe Auguste ${ }^{21}$, l'aire d'influence des évêques de Poitiers ${ }^{22}$, des vicomtes de Châtellerault ${ }^{23}$ ou de leurs voisins de Thouars ${ }^{24}$ fournissent le cadre d'enquêtes sur des séries de sites ou des espaces historiquement cohérents.

6. Bouvart 2003 et présentation dans le présent volume.

7. Durand 1989.

8. Gil 2000

9. Guyotjeannin 2000

10. Champagne 1997-1998.

11. Bourgeois 2000a et sous presse ; Cavaillès et Lecomte 2000 ; Châtelet-les Halles 1998.

12. Crépin-Leblond 1999.

13. Thèses en cours de Diane Joy et Thomas Rapin.

14. Germond, Champème, Fernandez 1987 et 1988.

15. Clairant 2001a et b.

16. Eneau 1990a et b; Debord et Dieudonné-Glad 1993; Bourgeois 2002 ; Ramirez 2002, Serdon 2003.

17. Ugé 1994 ; Lange 1995.

18. Grenouilloux, Migaud, Perez 1987; Grenouilloux 1988; Gilles 1991 ; Grenouilloux, Migaud 1993; Guintard 1993.

19. Léger 1999.

20. Baudry 2001b; Debord 2001.

21. Châtelain 1991.

22. Cherrier 1996 et 2004.

23. Gil 2000 ; Bourgeois 2000b.

24. Gautreau 1996 ; Jeanneau 2000. 


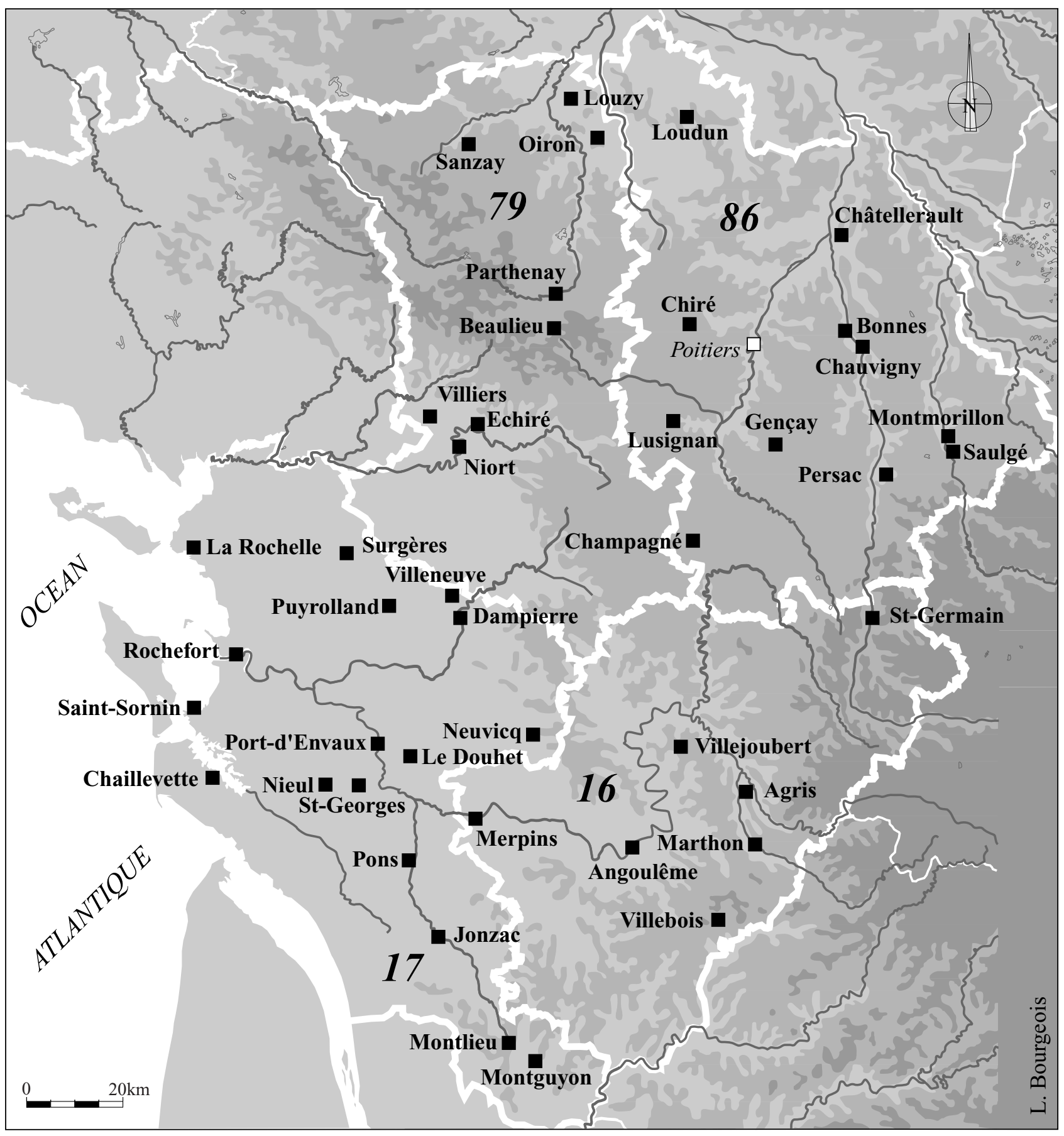

Archéologie des résidences aristocratiques médiévales en Poitou-Charentes :

opérations réalisées de 1987 à 2004. 


\section{Inventaire des opérations}

\section{Abréviations}

EV : évaluation

FP : fouille programmée

$\mathrm{PC}$ : projet collectif de recherches

PI : prospection inventaire

PT : prospection thématique
REL : opération de relevés

SD : sondage

$\mathrm{S}$ : fouille préventive

SP : sauvetage programmé

SU : sauvetage urgent

\begin{tabular}{|c|c|c|c|c|}
\hline DÉP. & COMMUNE/LIEU-DIT & TYPE & RESPONSABLE & DATES \\
\hline 16 & AGRIS, les Perrats & FP & B. Boulestin & $2002-2004$ \\
\hline 16 & ANGOULEME, îlot du Chapeau rouge & SU & J.-Ph. Baigl & 1994 \\
\hline 16 & ANGOULEME, îlot du Chapeau rouge & SP & J.-P. Nibodeau & 1995 \\
\hline 16 & MARTHON, le Château & SP & J.-Ph. Baigl & 1997 \\
\hline 16 & MARTHON, le Château & SD & F. Chiron & 1998 \\
\hline 16 & MARTHON, le Château & SD & G. Poitevin & 1999 \\
\hline 16 & MERPINS, Vieux Bourg & FP & B. Farago & 1987,1991 \\
\hline 16 & SAINT-GERMAIN-DE-CONFOLENS, le Château & EV & D. Brunie & 1997 \\
\hline 16 & SAINT-GERMAIN-DE-CONFOLENS, le Château & REL & $\begin{array}{l}\text { J.-C. Colin, } \\
\text { L. Prysmicki }\end{array}$ & 1998 \\
\hline 16 & VILLEBOIS-LAVALETTE, le Château & $\begin{array}{l}\text { SD } \\
\text { FP }\end{array}$ & A. Montigny & $\begin{array}{l}2003 \\
2004\end{array}$ \\
\hline 16 & $\begin{array}{l}\text { VILLEJOUBERT, la Garenne } \\
\text { (castrum d'Andone) } \\
\text { (ACR en cours depuis 2003) }\end{array}$ & $\begin{array}{l}\text { FP } \\
\text { SD } \\
\text { FP } \\
\text { SD }\end{array}$ & $\begin{array}{l}\text { A. Debord } \\
\text { L. Bourgeois }\end{array}$ & $\begin{array}{c}1987-1994 \\
1995 \\
2003 \\
2004\end{array}$ \\
\hline 17 & CHAILLEVETTE, Château de Beauregard & EV & K. Robin & 1999 \\
\hline 17 & DAMPIERRE-SUR-BOUTONNE, le Château & SD & A. Brillaud & 1988 \\
\hline 17 & DOUHET (le) & EV & J.-P. Nibodeau & 2004 \\
\hline 17 & JONZAC, Place du Château & EV & J.-P. Nibodeau & 2001 \\
\hline 17 & MONTGUYON, le Bourg & SD & B. Réaud & 1998 \\
\hline 17 & MONTLIEU-LE-GARDE & EV & N. Thomas & 1998 \\
\hline 17 & NEUVICQ-LE-CHATEAU, le Bois du Fouet & SD & J.-P. Cochon & 1992 \\
\hline 17 & NIEUIL-LES-SAINTES, le Logis & $\mathrm{EV}$ & J.-P. Cochon & 1992 \\
\hline 17 & PONS, le Château & SU & L. Lassarade & 1988 \\
\hline 17 & PORT-D’ENVAUX, le Bois muré & SD & J.-P. Nibodeau & 1994 \\
\hline 17 & PUYROLLAND, Charraud & EV & A. Bolle & 2001 \\
\hline 17 & ROCHEFORT, Jardin de la Marine & SP & Ph. Duprat & 1996 \\
\hline 17 & ROCHELLE (LA), place de Verdun & EV & A.-M. Fourteau & 1989 \\
\hline 17 & ROCHELLE (LA), place de Verdun & SP & A. Bocquet & $1994-1995$ \\
\hline 17 & ROCHELLE (LA), place de Verdun & EV & J.-Ph. Baigl & 1996 \\
\hline 17 & ROCHELLE (LA), place de Verdun & SP & J.-P. Nibodeau & 1998 \\
\hline 17 & SAINT-GEORGES-DES-COTEAUX, Cerveau & SD & A. Dalençon & 1999 \\
\hline 17 & SAINT-SORNIN, tour de Broue & SD & N. Faucherre & 2002 \\
\hline 17 & SURGERES, le château & $\mathrm{EV}$ & E. Normand & 2003 (2 autor.) \\
\hline 17 & VILLENEUVE-LA-COMTESSE, château & SD & J.-F. Buisson & 1991 \\
\hline 79 & BEAULIEU-SOUS-PARTHENAY, la Guyonnière & SD & A. Verdon & 1990 \\
\hline 79 & ECHIRE, le Coudray-Salbart & & E. Henry & 1990 \\
\hline 79 & ECHIRE, le Coudray-Salbart & SD & Ch. Maret & 1993 \\
\hline 79 & LOUZY, le Bourg & SU & J.-P. Nibodeau & 1990 \\
\hline 79 & NIORT, Espace niortais & SD & A. Bocquet & 1995 \\
\hline 79 & NIORT, Espace niortais & PR & J.-P. Nibodeau & 1999 \\
\hline 79 & NIORT, îlot de Vaize & $\begin{array}{c}\text { REL } \\
\mathrm{S}\end{array}$ & $\begin{array}{l}\text { L. Prysmicki } \\
\text { D. Brunie }\end{array}$ & $\begin{array}{l}2002 \\
2002\end{array}$ \\
\hline
\end{tabular}




\begin{tabular}{|c|c|c|c|c|}
\hline 79 & NIORT, place du Donjon & $\mathrm{EV}$ & E. Henry & 1991 \\
\hline 79 & OIRON, le Château & EV & A. Bocquet & 1991 \\
\hline 79 & OIRON, le Château & SU & K. Robin & 1997 \\
\hline 79 & OIRON, le Château & $\begin{array}{l}\mathrm{EV} \\
\mathrm{SU}\end{array}$ & Th. Cornec & $2000-2002$ \\
\hline 79 & OIRON, le grand Bois & SD & G. Germond & 1987 \\
\hline 79 & PARTHENAY, fortifications & $\mathrm{PC}$ & M. Cavaillès, M.-P. Baudry & $1995,1997-1998$ \\
\hline 79 & PARTHENAY, le Château & $\begin{array}{l}\text { SU } \\
\text { SD, SU } \\
\text { FP }\end{array}$ & $\begin{array}{l}\text { A. Verdon } \\
\text { M. Cavaillès }\end{array}$ & $\begin{array}{c}1991 \text { (3 autor.) } \\
\text { 1989, 1991, } 1996 \text { (2 } \\
\text { autor.), 1998, } 2000 \\
2002\end{array}$ \\
\hline 79 & PARTHENAY, le Château & SU, SP & A. Verdon & $\begin{array}{l}1987-1988 \\
1990,1992\end{array}$ \\
\hline 79 & PARTHENAY, le Château & SD & L. Fleuret & 1996 \\
\hline 79 & SANZAY, le Château & SD & M.-C. Bakkal-Lagarde & $1998,2000,2001,2003$ \\
\hline 79 & VILLIERS-EN-PLAINE, la Vallée de Faye & SP & A. Bolle & 1999 \\
\hline 86 & BONNES, Touffou & $\mathrm{S}$ & M. Aubrun & 1992 \\
\hline 86 & CHAMPAGNE-LE-SEC, maison noble & $\mathrm{S}$ & Ph. Blonde & 1991 \\
\hline 86 & CHATELLERAULT (vicomté de) & PI & V. Gil & 2000 \\
\hline 86 & CHATELLERAULT, Pouthumé & S & Th. Cornec & 2003 \\
\hline 86 & CHAUVIGNY, château baronnial & $\mathrm{SU}$ & I. Bertrand, Th. Eneau & 1993 \\
\hline 86 & CHAUVIGNY, château de Montléon & & Th. Eneau & 1989-90, 1992 \\
\hline 86 & CHAUVIGNY, donjon de Gouzon & SU & Th. Eneau & 1987-88, 1990 \\
\hline 86 & CHIRE-EN-MONTREUIL, le Château & $\begin{array}{l}\text { SU } \\
\text { FP }\end{array}$ & D. Vivier & $\begin{array}{l}1996-1998 \\
2001,2002\end{array}$ \\
\hline 86 & GENCAY, le Vieux Château & SU & J.-P. Cochon, J.-P. Nibodeau & 1991 \\
\hline 86 & GENCAY, le Vieux Château & SU & J.-P. Nibodeau & 1993,1996 \\
\hline 86 & GENCAY, le Vieux Château & SU & L. Bourgeois & 1997 \\
\hline 86 & GENCAY, le Vieux Château & $\mathrm{SU}$ & L. Prysmicki & 1997 \\
\hline 86 & LOUDUN, le Château & REL & B. Favreau & 1998 \\
\hline 86 & LUSIGNAN, le Château & EV & J.-P. Nibodeau & 1996 \\
\hline 86 & LUSIGNAN, le Château & $\mathrm{EV}$ & L. Bourgeois & 1998 \\
\hline 86 & MONTMORILLON, la Motte & SU & J.-P. Cochon & 1991 \\
\hline 86 & PERSAC, la Mothe & SD & Y. Comte & 1991 \\
\hline 86 & SAULGE, les Grands Bois & SD & Ph. Durand & 1996 \\
\hline
\end{tabular}




\section{Bibliographie régionale}

Allard T., J. Debelle, N. Faucherre 2002 : Le château de Touffou (Vienne), La Crèche, Geste Editions.

Andrault J.-P., M.-F. Meunier 1998 : Châteaux et gentihommières du HautPoitou, Paris, Nouvelles éditions latines.

Araguas Ph. 1999: "Le château de la Léotardie, commune de Nonac [Charente] ", Congrès archéologique de France, $153^{e}$ session, 1995, Charente, Paris, Société française d'archéologie, 293-298.

Archéologie de la France 1989: Archéologie de la France: 30 ans de découvertes, catalogue de l'exposition, Paris, RMN, 396-399.

Association Promotion-Patrimoine 1991 : Châteaux, manoirs et logis: les Deux-Sèvres, Niort, Association Promotion Patrimoine.

Association Promotion-Patrimoine (1993a) : Châteaux, manoirs et logis : la Charente-Maritime. Niort : Association Promotion-Patrimoine.

Association Promotion-Patrimoine (1993b) : Châteaux, manoirs et logis : la Charente. Chauray: Patrimoines et Médias.

Audebrand F. 2004: "Le château de Coulonges-sur-l'Autize", Congrès archéologique de France. Monuments des Deux-Sèvres, 159 session, 2001, Société française d'archéologie, Paris, 2004, 141-157.

Autrant F. 1999 : "La tour Maubergeon à Poitiers : un monument de paix ?", Faire mémoire : souvenir et commémoration au Moyen Age $(\mathrm{Cl}$. Carozzi et H. Taviani-Carozzi dir.), Aix-en-Provence, Publications de l’Université de Provence, 9-13

Bakkal-Lagarde M.-C. 1991 : "Château de l'Isle à la Crèche [Deux-Sèvres]", Bull. ADANE, n 3, 60-62.

Bakkal-Lagarde M.-C. 2000 : " Résultats des premiers sondages archéologiques au château de Sanzay (Deux-Sèvres) ", Bull. AAPC, n 29, 67-69.

Baudry M.-P. 1989 : Le château du Coudray-Salbart. mém. maîtr. d'histoire de l'art et d'archéologie (L. Pressouyre dir.), Univ. Paris I-Sorbonne, 2 vol.

Baudry M.-P. 1991 : " Le château du Coudray-Salbart”. Bull . archéol. du CTHS Antiquités nationales, fasc. 23-24, 137-212.

Baudry M.-P. 1993 : “Les donjons de Loudun ”. Bull. Soc. amis Pays lochois t. $9,163-169$

Baudry M.-P. 1999 : "Les châteaux des Lusignans en Poitou : 1154-1242", Isabelle d'Angoulême, comtesse-reine et son temps (1186-1246). Actes du colloque de Lusignan, 1996, Poitiers, CESCM, 136-146 et pl. XIX-XXI.

Baudry M.-P. 2000 : “Les fortifications des Plantagenêt à Thouars ", La cour Plantagenêt (1154-1204) (M. Aurell dir.), Poitiers, CESCM, 297-314.

Baudry M.-P. 2001a : "Le château des Plantagenêt à Niort", Les fortifications des Plantagenêt (M.-P. Baudry dir.), Poitiers, CESCM, 23-40.

Baudry M.-P. 2001b : Les fortifications des Plantagenêt en Poitou (1154-1242), Paris, éditions du CTHS, 383 p. (Mémoires de la section d'archéologie et d'histoire de l'art, 11).

Baudry M.-P. 2002 : “ Les fortifications de la ville, du château et de l'abbaye de Saint-Maixent", Le château et la ville : conjonction, opposition, juxtaposition (XIe-XVIIIe siècle) : Actes du 125e congrès national des sociétés historiques et scientifiques (G. Blieck et coll. dir.), Lille, 2000, Paris, éd. du CTHS, 21-32.

Baudry M.-P. 2004a : “Les 'châteaux d'Aliénor': palais, fortifications ou prisons ", 303, n spécial Aliénor d'Aquitaine, 118-127.

Baudry M.-P. 2004b : "Le Coudray-Salbart: un château royal ?", Congrès archéologique de France. Monuments des Deux-Sèvres, $159^{e}$ session, 2001, Société française d'archéologie, Paris, 2004, 131-139.

Baudry M.-P. 2004c: "Le donjon de Niort ", Congrès archéologique de France. Monuments des Deux-Sèvres, 159 session, 2001, Société française d'archéologie, Paris, 2004, 193-199.

Baudry M.-P. et B. Fillon 2004: "Le château de Bressuire", Congrès archéologique de France. Monuments des Deux-Sèvres, 159 session, 2001, Société française d'archéologie, Paris, 2004, 83-98.

Baudry M.-P., K. Lebreton-Royer 1999 : “Le château de Villebois-Lavalette du Xe au XIIIe siècle ”, Congrès archéologique de France, 153e session, 1995. Charente, Paris, Société française d'archéologie, 349-356.

Bercé Y.-M. 1996 : “ Les politiques de démantèlement de châteaux ”, Châteaux et pouvoirs, Xe-XIXe siècles, Bordeaux, 121-131.

Bériac F., S. Cauchois 1998: "Garnisons, voies de communications et ravitaillement en 1324-1325: les troupes anglo-gasconnes dans le duché d'Aquitaine ", Châteaux, routes et rivières. Actes des rencontres d'archéologie et d'histoire du Périgord, 26-28 septembre 1997 (F. Bériac, A.-M. Cocula dir.), Bordeaux, 45-71 [pour Saintes et Montendre].

Bernage G. 2001 : "Le Coudray-Salbart, une étonnante forteresse du XIIIe siècle ", Moyen Age, n 22, juin 2001, 16-31.

Bernage G. 2002 : "Cherveux, château d'un capitaine de la garde écossaise ", Moyen Age, n 31, nov.-déc. 2002, 12-28.

Bernage G. 2003 : “Le château de Tennessus ”, Moyen Age, n 32, janv.-févr. 2003, 30-36.

Beving J. 1997 : Le château de Château-Larcher [Vienne], mém. maîtr. d'archéologie (N. Dieudonné-Glad dir.), Univ. Poitiers.

Blomme Y. 1993 : Poitou gothique, Paris, Picard.

Bocquet A., P. Mille, J.-P. Nibodeau 2000 : “ Découvertes archéologiques sur le château de La Rochelle ", Les fortifications dans les domaines Plantagenêt, XIIe-XVe siècles (M.-P. Baudry dir.), actes du colloque de Poitiers, 1994, Poitiers, CESCM, 117-118.

Bonnin J.-C. 1999 : “ Documents sur le château et le prieuré de Rochefort (13231450) ", Roccafortis, n²3, 23-25.

Bonnin J.-C. 1996 : L'ancien château de Vauclerc à La Rochelle, La Rochelle.

Bourdonneau L. 1998 : Les châteaux du Poitou dans les sources écrites des XIVe et XVe siècles, mém. maîtr. d'histoire (G. Pon dir.), Univ. Poitiers.

Bourgeois L. dir. 2000a : Les petites villes du Haut-Poitou de l'Antiquité au Moyen Age: formes et monuments, t. 1. : Bressuire, Brioux-sur-Boutonne, Loudun, Montmorillon, Saint-Savin-sur-Gartempe, Thouars (L. Bourgeois dir.), Chauvigny, APC.

Bourgeois L. 2000b : "Du Vieux-Poitiers à Châtellerault : le confluent de la Vienne et du Clain de l'Antiquité au Moyen Age”, Bull. Soc. Antiq. Ouest, $5^{\mathrm{e}}$ s., t. XIV, 163-194.

Bourgeois L. 2001: “Le castrum d'Andone: orientation des nouvelles recherches", Bull. AAPC, n 30, 87-89.

Bourgeois L. 2002 : “Pièces de jeu des Xe-XIIe s. et milieu aristocratique dans le Centre-Ouest de la France ", Aquitania, 18 (paru en 2003), 373-400.

Bourgeois L. dir. (sous presse) : Les petites villes du Haut-Poitou de l'Antiquité au Moyen Age: formes et monuments, t. 2 : Angles-sur-l'Anglin, ArgentonChâteau, Charroux, Melle, Rom, Parthenay (L. Bourgeois dir.), Chauvigny, APC.

Bouvard P. 2003 : Le château de Chiré-en-Montreuil (Deux-Sèvres) : étude archéologique des élévations, mém. maitr. d'archéologie (L. Bourgeois dir.), Univ. Poitiers, 2 vol.

Brillaud A. 1989 : "Découvertes archéologiques au château de Dampierre-surBoutonne [Charente-Maritime] ", Bull. monum., t. 147, fasc. III, 247-248.

Bruand O. 2001 : "Les qualificatifs de palatium, castrum et castellum sur les monnaies mérovingiennes et carolingiennes", Aux marches du palais (A. Renoux dir.), actes du VIIe congrès international d'archéologie médiévale (Le Mans, 1999), Le Mans, 23-28.

Bucherie L. dir. 1993 : Les graffiti du château de François Ier à Cognac, Segonzac.

Cavaillès M. 2004 : "Le château de Parthenay", Congrès archéologique de France. Monuments des Deux-Sèvres, $159^{e}$ session, 2001, Société française d'archéologie, Paris, 2004, 217-227.

Cavaillès M., M.-P. Baudry-Parthenay 1998 : "Parthenay, place forte du Poitou ", Archeologia, n 347, juillet-août 1998, 34-45.

Cavaillès M., N. Lecomte 2000 : Parthenay, Paris, Editions du Patrimoine (Document d'évaluation du patrimoine archéologique des villes de France; 19).

Champagne A. 1997-98 : "Une reconstruction du XVe s. en Poitou : financement et approvisionnement en matériaux du chantier de Vasles [Vienne] ". Aquitania, t. $15,287-307$.

Champagne A. 2001 : "Un petit seigneur poitevin et la reconstruction en Poitou au XVe s. : le chantier de Vasles ", Du projet au chantier : maîtrise d'ouvrage et maitrise d'œuvre du XIVe au XVIe s. (O. Chapelot dir.), actes du colloque de Vincennes, $1^{\text {er-3 }}$ octobre 1998, Paris, EHESS, 197-217.

Champagne A. (sous presse) : " Pouvoir seigneurial et habitat rural au XVe s. en Centre-Ouest", Maison rurale et habitat dispersé : des origines aux années 1960, actes du colloque de l'université de Rennes II, 29-31 mai 2002. 
Champeme L.-M. 1990 : "Sites médiévaux et photographies aériennes dans le département des Deux-Sèvres : premières conclusions ", Sites défensifs et sites fortifiés au Moyen Age entre Loire et Pyrénées, actes du premier colloque d'Aquitania, 1987, Bordeaux, 163-171 (Supplément 4 d'Aquitania).

Chapacou D. 1989 : "La motte féodale du Breuil à La Villedieu [CharenteMaritime]", Bull. Assoc. archéol. hist. Aulnay, t. 6, 19-20.

Charraud J.-R. 1987 : “ Un petit fief : le logis de Sainte-Terre, paroisse de Benay [Benest, Charente] ", Bull. Mém. Soc. archéol. hist. Charente, fasc. 2, 119-131.

Chasseboeuf F. 1995 : "La demeure seigneuriale en Angoumois et en Saintonge au temps de François Ier ", Actes du colloque du 500 anniversaire de Françoi Ier (Cognac, 1994), Annales du GREH, n 16, 42-74.

Chatelain A. 1991: "Recherches sur les châteaux de Philippe Auguste", Archéol. méd., t. XXI, 115-161.

Chauveaud J. 1993 : " Où était le logis de Puychaussac [Aussac, Charente] ?" Bull. Mém. Soc. archéol. hist. Charente, 210-212.

Cherrier N. 1996 : “ Châteaux, frontières et espaces forestiers à l'est du Poitou du Xe au début du XIIIe s. ”, Château-Gaillard, XVII, 1994 (1996), 45-52.

Cherrier-Lévêque N. 2004: “Les châteaux des seigneuries d'Angles et Chauvigny (diocèse de Poitiers) dans les guerres du XIIe au XVe s. ", Châtean et guerre (éd. M. Combert et R. Hérin), actes des rencontres d'archéologie et d'histoire en Périgord, 25-28 septembre 1998, Bordeaux, 11-27.

Ciukaj C. 1997: Les châteaux-forts en Charente-Maritime au XVe s. (bibliographie, état de la question), mém. DEA (M.-Th. Camus dir.), Univ. Poitiers.

Clairant A. 2001a : "Le trésor de Taillebourg (Charente-Maritime) : un dépôt monétaire de 215 monnaies noires, enterré entre 1432 et 1436 ", Trésors monétaires, XIX, 195-218.

Clairant A. 2001b : " Les monnaies des fouilles de Parthenay (Deux-Sèvres) et de Gençay (Vienne) ”, Trésors monétaires, XIX, 289-348.

Collectif 1992: Le château des seigneurs de Parthenay, Chauvigny, APC (Mémoire VIII)

Collectif 1993: Châteaux, logis et demeures anciennes de la Charente, Angoulême, éd. Bruno Sépulchre.

Collectif 1998 : Châtelet-les-Halles : 1000 ans d'histoire urbaine à Angoulême (Charente), Angoulême.

Corvisier Chr. 1998 : Les grosses tours de plan circulaire ou centré en France avant 1200. Etude sur les antécédents de la politique castrale de PhilippeAuguste, thèse nouveau régime (L. Pressouyre dir.), Univ. Paris I-Sorbonne.

Corvisier Chr. 1999: "Le château de Bayers [Charente]". Congrès archéologique de France, 153e session, 1995, Charente, Paris, Société française d'archéologie, 111-123.

Crépin-Leblond Th. 1999: "Le palais épiscopal d'Angoulême”, Congrès archéologique de France, 153e session, 1995, Charente, Paris, Société française d'archéologie, 27-35.

Czajkowski C. 1999: Le château de Taillebourg [Charente-Maritime] des origines au XVe s., mém. maîtr. d'histoire de l'art (N. Dieudonné-Glad et N Faucherre dir.), Univ. Poitiers.

Dalancon A., D. Baurand 1999 : "Saint-Georges-des-Côteaux [CharenteMaritime], 'Cerveau', château de Cerveau ", Bull. Soc. archéol. hist. CharenteMaritime, t. 26, p. 53-66.

Debord A. 1987 : " Châteaux et bourgs castraux charentais au Moyen Age ”, Bull. liaison Soc. arch. hist. Charente-Maritime, $\mathrm{n}^{\circ} 14,8-9$.

Debord A. 1988 : “L'église, le château et l'organisation de l'espace dans l'Ouest de la France, Xe-XIVe s. ", L'église et le château, Xe-XVIIIe s. Cahiers de Commarque, 27-43.

Debord A. 1989 : “Villejoubert, castrum d'Andone (16)", Romains et barbares entre Loire et Gironde, IVe-Xe s., catalogue d'exposition, Poitiers, 164.

Debord A. 1990 : “ La recherche en matière de fortifications médiévales dans la région Poitou-Charentes : bilan et perspectives ", Sites défensifs et sites fortifiés au Moyen Age entre Loire et Pyrénées, actes du premier colloque d'Aquitania 1987, Bordeaux, 151-161 (Supplément 4 d'Aquitania)

Debord A. 1991 : «Naissance, évolution et fonctions du château médiéval ", Les archéologiques Poitou-Charentes, Poitiers 23-24 novembre 1991. Bilans et perspectives de la recherche régionale. Bull. AAPC, 167-172.

Debord A. 1992 : "Confort et inconfort dans les châteaux du Xe au XIIe s.", Actes des IVe rencontres internationales d'arch. et d'hist. de Commarque, septembre 1990, 11-27.
Debord A. 1994 : “Andone (Charente, c. Villejoubert), résidence et forteresse du comte d'Angoulême", Palais médiévaux (France, Belgique) : 25 ans d'archéologie (A. Renoux dir.), Le Mans, Publications de l'Université du Maine, 17-18.

Debord A. 1995: "Une résidence comtale de l'an Mil: Andone" et "Le mobilier recueilli sur le castrum d'Andone", Splendeurs de Saint-Martial de Limoges au temps d'Adémar de Chabannes (B. Barrière, G. Cantié, V. Notin dir.), catalogue de l'exposition, Limoges, Musée de l'Evêché, 33-36 et 41-42.

Debord A. 1998 : "Le château et le ban : mainmise sur l'espace et les hommes dans le royaume de France (Xe-XIIIe s.)", Château et pouvoir, Bordeaux, 3-17.

Debord A. 2000 : Aristocratie et pouvoir: le rôle du château dans la France médiévale, Paris, Picard, (Espaces médiévaux).

Debord A. 2001 : " La politique de fortification des Plantagenêt dans la seconde moitié du XIIe s. ”, Les fortifications dans les domaines Plantagenêt, XIIe-XIVe siècles, Poitiers, CESCM, 9-14.

Debord A. 2003 : "Châteaux et villages dans l'Ouest aquitain (XIe-XIIIe s.)", Château et village, actes des $2 \mathrm{e}$ rencontres internationales d'archéo. et d'hist. du Périgord (Périgueux, 1995), Bordeaux, 9-18.

Debord A., N. Dieudonné-Glad 1987 : "Andone (Charente) : étude structurale d'outils et d'armes du Xe s. Apports à la connaissance de l'artisanat du fer ", Archéo. Méd., t. XXIII, 107-129.

Desquesnes R. et coll. 1993 : Les fortifications du littoral : la Charente-Maritime, Chauray, éd. Patrimoines et Médias.

Didier F. 1999 : "Le château de Villebois-Lavalette du XVe au XXe siècle : de la forteresse à la résidence ", Congrès archéologique de France, $153^{e}$ session, 1995, Charente, Paris, Société française d'archéologie, 357-369.

Drapeau A. 2001 : Le château de Dissay [Vienne] : état actuel et essais de restitution à l'époque de Pierre d'Amboise (1481-1505), mém. maitr. d'histoire de l'art (E. Thomas dir.), Univ. Poitiers, 3 vol.

Dubourg-Noves P. 1999 : “ Le château d'Angoulême et les donjons polygonaux des Lusignan ", Isabelle d'Angoulême, comtesse-reine et son temps (11861246), actes du colloque de Lusignan, 8-10 novembre 1996, Poitiers, CESCM, 177-183 et pl. XXX et XXXI.

Duguet J. 1998 : “Le 'château’ du Breuil dans la paroisse de Saint-Symphorien [Charente-Maritime] ", Roccafortis, $\mathrm{n}^{\circ}$ 20, 270.

Dumas-Delage S. 2004 : « Une tour maîtresse à contreforts au château de SaintGermain-de-Confolens (Charente)", Tours seigneuriales de l'Ouest : travaux récents sur quelques tours maîtresses, de la Normandie à la Catalogne $(\mathrm{N}$. Faucherre et Chr. Rémy éd.), Poitiers, CESCM, 87-92.

Duprat Ph. 1997 : “Le site médiéval du Jardin de la Marine à Rochefort", Roccafortis, $\mathrm{n}^{\circ} 19,170-183$.

Duprat Ph., F.-Y. Le Blanc 1999 : “Nouvelles données sur l'enceinte fortifiée médiévale de Rochefort", Roccafortis, $n^{\circ} 24,75-83$.

Durand Ph. 1987 : "Les châteaux en Haut-Poitou ", Le paysage monumental autour de l'an mil (X. Barral i Altet, dir.), Paris, Picard.

Durand Ph. 1989 : “L'entrée du petit château du XVe s. à travers l'exemple du Montmorillonnais ", Actes du $3^{e}$ colloque de castellologie de Flaran, s.l.

Durand Ph. 1997 : “Le château d'Angles-sur-l'Anglin ”, Bull. Soc. amis Pays lochois, 107-118.

Durand Ph. 1999a : Le château fort, Bordeaux, éd. Gisserot [nombreux exemples dans la Vienne].

Durand Ph. 1999b : "Le château de La Rochefoucauld : le Moyen Age", Congrès archéologique de France, $153^{e}$ session, 1995, Charente, Paris, Société française d'archéologie, 217-230.

Durand Ph. 1999c: "Le châtelet d'Angoulême: aspect archéologique et hypothèse de datation ", Isabelle d'Angoulême, comtesse-reine et son temps (1186-1246), actes du colloque de Lusignan, 8-10 novembre 1996, Poitiers, CESCM, 161-171 et pl. XXV à XXVIII.

Durand Ph. 2001a: "Les fortifications de Poitiers : étude et hypothèse de datation des éléments subsistants", Les fortifications dans les domaines Plantagenêt, XIIe-XVe s., Poitiers, 61-76.

Durand Ph. 2001b : "Le château de Moncontour (Vienne)", Bull. Soc. amis Pays Lochois, $\mathrm{n}^{\circ}$ 17, 2001, p. 141-152.

Durand Ph., J.-P. Andrault dir. 1995 : Châteaux, manoirs et logis : la Vienne, Chauray, éd. Patrimoines et Médias.

Eneau Th. 1989 : "Fouilles au donjon de Gouzon ", Le Pays chauvinois, 27, 44-45.

Eneau Th. 1990a : "Chauvigny, château de Montléon, mobilier fin XVIe s.", Cahiers du pays chauvinois, $6,24 \mathrm{p}$ 
Eneau Th. 1990b : "Le site du château de Montléon : fosse dépotoir et mobilier de la fin du XVIe s. «, Le Pays chauvinois, 33-56.

Evrard Chr. 1992a : Le château médiéval de Gençay (Vienne) : histoire et architecture, Poitiers, Association des Amis du Vieux-Château de Gençay.

Evrard Chr. 1992b : Les ouvrages d'entrée fortifiés des châteaux du Poitou, mém. DEA (M.-Th. Camus dir.), Univ. Poitiers.

Faucherre N. 1993 : Note sur l'intérêt monumental de la grosse tour du château de Monthoiron [Vienne], août 1993 [dossier CRMH Poitou-Charentes].

Faucherre N. 2001 : "L'étude de la tour du comte Jean au château de Cognac", Bull. monum., t. 159, fasc. 1, 41-42.

Faucherre N. 2004a : "La tour de Broue (Charente-Maritime) : l'oeuvre du comte d'Anjou ", Tours seigneuriales de l'Ouest : travaux récents sur quelques tours maîtresses, de la Normandie à la Catalogne (N. Faucherre et Chr. Rémy éd.), Poitiers, CESCM, 69-74.

Faucherre N. 2004b : "Touffou (Vienne) : la tour dédoublée ", Tours seigneuriales de l'Ouest: travaux récents sur quelques tours maîtresses, de la Normandie à la Catalogne (N. Faucherre et Chr. Rémy éd.), Poitiers, CESCM, 65-68.

Faucherre N. 2004c : "Le château de Cherveux ", Congrès archéologique de France. Monuments des Deux-Sèvres, 159e session, 2001, Société française d'archéologie, Paris, 2004, 121-129.

Faucherre N. et A. Pellerin 2003 : Crazannes, logis alchimique, Le Croît vif, Paris.

Faucherre N., S. Servant 2004 : "Pons (Charente-Maritime) : la force du symbole de 1240 à 1900 ", Tours seigneuriales de l'Ouest : travaux récents sur quelques tours maîtresses, de la Normandie à la Catalogne (N. Faucherre et Chr. Rémy éd.), Poitiers, CESCM, 75-86.

Forgeaud D. 1987 : “ Le château, le châtelet, la petite halle et la porte Chandos [à Angoulême]", Bull. Soc. archéol. hist. Charente, 174-200.

Gautreau C. 1996 : Les châteaux de la vicomté de Thouars : état de la question, mém. DEA (M.-Th. Camus dir.), Univ. Poitiers, 2 vol.

Germond G., L.-M. Champeme, L. Fernandez 1987: "Le problème archéologique des garennes: la contribution du grand Bois à Oiron (DeuxSèvres) ", Bull. Soc. hist. scien. Deux-Sèvres, 2e s., t. XX, 189-196.

Germond G., L.-M. Champeme, L. Fernandez 1988: "Le problème archéologique des garennes ", Archéo. méd., t. 18, 239-254.

Gil V. 1997 : Monographie du château de Berrie, mém. maîtr. (M.-Th. Camus dir.), Univ. Poitiers.

Gil V. 2000 : Inventaire des sites castraux de l'ancienne vicomté de Châtellerault des origines à 1514, mém. DEA (Cl. Andrault-Schmitt et M.-Th. Camus dir.), Univ. Poitiers, 3 vol.

Gil V. et Ph. Luçon 2004 : "Le château de Glénay ", Congrès archéologique de France. Monuments des Deux-Sèvres, $159^{e}$ session, 2001, Société française d'archéologie, Paris, 2004, 159-170.

Gilles R. 1991 : L'avifaune d'Andone aux Xe-XIe s., mém. DEA, Univ. Paris ISorbonne.

Grenouilloux A. 1988: "Restauration du poids initial des ossements archéologiques des bovins par une étude métrique des métatarsiens: application à l'évaluation du poids des bovins du site médiéval d'Andone ", Revue d'archéométrie, XII, 41-55.

Grenouilloux A., Ph. Migaud 1993 : “ Sauvages, commensaux ou domestiques : l'animal dans l'économie alimentaire d'un castrum de l'an Mil ", L'homme l'animal domestique et l'environnement du Moyen Age au XVIIIe s. (R. Durand dir.), Nantes, Publications de l'Université de Nantes, 163-176.

Grenouilloux A., Ph. Migaud, M. Perez 1987 : Répartition des restes osseux animaux sur le site d'Andone, Pouzauges, Centre d'études zoo-archéologiques de l'Ouest.

Grimbert L. 2000 : “Les fouilles du bastion à Saintes (17) ”. Bull. Soc. archéol. hist. Charente-Maritime, t. 27, 81-91.

Guénégan J. 1993 : “ L'enceinte circulaire de Fiole, c. de Champagne [CharenteMaritime] ", Roccafortis, $\mathrm{n}^{\circ} 12,168$.

Guillaume J. 2004 : “Oiron au XVIe s. : le château et l'église ”, Congrès archéologique de France. Monuments des Deux-Sèvres, $159^{e}$ session, 2001, Société française d'archéologie, Paris, 2004, 201-207.

Guintard Cl. 1993 : Analyse de fragments de mâchoires de porcs (site d'Andone), Nantes, Ecole vétérinaire, laboratoire d'anatomie des animaux domestiques.

Guyonnet B. 1997 : Les petites villes de la Marche limousine, mém. DEA de civilisation médiévale (M.-Th. Camus dir.), Univ. Poitiers.
Guyotjeannin O. 2000: "Les autorisations royales de fortifier enregistrées en chancellerie (1441-1497)", Guerre, pouvoir et noblesse: mélanges en l'honneur de Philippe Contamine (J. Paviot et J. Verger dir.), Paris, Presse de l'Université Paris-Sorbonne, 343-352.

Jeanneau C. 1996 : La mutation castrale en Poitou de 965 à 1030, mém. maîtr. d'histoire (R. Favreau dir.), Univ. Poitiers.

Jeanneau C. 2000a : La construction des châteaux et la «mutation féodale»: les transformations de la société en Bas-Poitou (1000-1150), mém. DEA de civilisation médiévale (M. Aurell dir.), Univ. Poitiers.

Jeanneau C. 2000b : “La mutation castrale et les transformations de la société en Poitou aux Xe et XIe s. ”, Bull. hist. archéol. Pays thouarsais, 29-45.

Joy D. 2000 : “ Le gothique international à Poitiers : les oeuvres sculptées pour Jean de Berry à la fin du XIVe s. ”, Bull. Soc. Antiq. Ouest, 5e s., t. XIV (paru en 2002), 318 .

Joy D. 2000 : Autour de la sculpture du Palais de Poitiers : la sculpture du gothique tardif en Poitou, mém. DEA de civilisation médiévale $(\mathrm{Cl}$. Andrault dir.), Univ. Poitiers.

Lange A. 1995 : "Les carreaux de pavage en terre vernissée du Moyen Age découverts au cours des fouilles du château du Vieux Bourg et de l'abbaye de La Frenade à Merpins [Charente] ", Bull. Institut hist. archéol. Cognac et Cognaçais, t. $6, \mathrm{n}^{\circ}$ 5, 9-18.

Lange A. 1997 : " Le manoir du Maine-Giraud à Champagne-Vigny (Charente) ", Bull. Institut hist. archéol. Cognac et Cognaçais , t. 7, n $2,19-22$.

Langeuin P. 2000 : " La couverture de la tour double du Coudray-Salbart ", Les fortifications dans les domaines Plantagenêt (XIIe-XIVe s.) (M.-P. Baudry dir.), actes du colloque de Poitiers, 1994, Poitiers, CESCM, 123-124.

Lassarade L. 1988 : “Fosses devant l'entrée du château de Pons”, Archéo. pontoise, $\mathrm{n}^{\circ} 84$, n. p.

Lebreton-Royer K. 1997 : Les chapelles castrales construites au Moyen Age en Charente, mém. maîtr. d'histoire de l'art (J. Lacoste dir.), Univ. Bordeaux III.

Léger J.-G. 1992 : "Mille ans d'histoire avec (sic) le château de Barbezieux", Bull. Soc. arch. hist. Barbezieux, t. XXXV, 51-112.

Léger S. 1999 : “Gabrielle de Bourbon : une grande dame de l'Ouest à la fin du Moyen Age. Etude de son cadre de vie à partir de l'inventaire après décès des biens demeurés au château de Thouars (1516)", Autour de Marguerite d'Ecosse : reines, princesses et dames au XVe s. (G. et Ph. Contamine dir.). Actes du colloque de Thouars (23-24 mai 1997), Paris, Champion, 181-200 (Etudes d'histoire médiévale ; 4).

Luçon Ph. 1997 : Le château de Hérisson (79), mém. maîtr. (M.-Th. Camus dir.), Univ. Poitiers, 2 vol.

Luçon Ph. 2004 : "Le château de Tennessus", Congrès archéologique de France. Monuments des Deux-Sèvres, $159^{e}$ session, 2001, Société française d'archéologie, Paris, 2004, 305-310.

Macé L. 1993 : “ Le site archéologique de la Vergne [motte, c. de Sainte-Gemme, Charente-Maritime] ", Roccafortis, $\mathrm{n}^{\circ}$ 12, 142-151.

Macé S. 1998 : Château du Haut-Clairvaux, chapelle Notre-Dame-des-Vergers, mém. maîtr. d'histoire de l'art (M.-Th. Camus dir.), Univ. Poitiers, 4 vol.

Maltat B., G. Voisin 2002 : "Les vestiges du château de Tourriers [Charente]", Jadis [Saint-Amant-de-Boixe], $\mathrm{n}^{\circ}$ 1, 40-42.

Martin-Civat P. 1995 : "Le château royal de Cognac [réédition de l'édition de 1972-1973] ”, Bull. Institut hist. archéol. Cognac et Cognaçais, t. 6, nº 5, 19-49.

Martins 2000 : Le château de Marthon (Charente), mém. DEA d'histoire de l'art (Ph. Durand dir.), Univ. Bordeaux III.

Mesqui J. 2000 : "Les tours à archères dans le domaine Plantegenêt français, 1160-1205", Les fortifications dans les domaines Plantagenêt (XIIe-XIVe s.) (M.-P. Baudry dir.), Poitiers, CESCM, 77-88.

Michaud F. 1994 : Le château d'Angles-sur-l'Anglin (Vienne), mém. maîtr. d'histoire de l'art (M.-Th. Camus dir.), Univ. Poitiers, 2 vol.

Migaud Ph. 1989 : "Une première approche du profil céphalique des suinés sur le site d'Andone (Saint-Amand-de-Boixe, Charente) ", Anthropozoologica, 10, 23-30.

Migaud Ph. 1991 : "Premiers résultats concernant l'étude de la cuisson des aliments sur le site d'Andone (Charente, Xe-XIe s.) ", Anthropozoologica, 14-15, 67-68.

Morhain A. 2004 : Le château de Fouras. Etude historique et architecturale, T.E.R. d'histoire médiévale et moderne (N. Faucherre dir.), Univ. La Rochelle.

Nibodeau J.-P. 1999 : "Les vestiges du Châtelet d'Angoulême", Isabelle d'Angouleme, comtesse-reine et son temps (1186-1246), actes du colloque de Lusignan, 8-10 novembre 1996, Poitiers, CESCM, 173-176 et pl. XXIX. 
Ollivier A. 1990 : "Sites médiévaux et photographie aérienne dans le nordouest du département de la Vienne ", Sites défensifs et sites fortifiés au Moyen Age entre Loire et Pyrénées, actes du premier colloque d'Aquitania (1987), Bordeaux, 173-176 (Supplément 4 d'Aquitania).

Petit J.-C. 2000-2001 : " Haut-Poitou : prospection sur les ouvrages souterrains ", Archéo. méd., 30-31, 507-511 [Bonnes et Queaux, Vienne].

Piboule P. 1990 : "Relations entre souterrains et fortifications : exemples en Poitou-Charentes", Sites défensifs et sites fortifiés au Moyen Age entre Loire et Pyrénées, actes du premier colloque d'Aquitania (1987), Bordeaux, 191-202 (Supplément 4 d'Aquitania).

Pilarski-Fourré E. 1996 : Le château de Ternay en Loudunais aux XVe et XVIIe s. [Vienne], mém. maîtr. d'histoire de l'art (M.-Th. Camus dir.), Univ. Poitiers.

Proust L. 1995 : Le château médiéval de Parthenay, mém. maîtr. d'histoire de l'art (M.-Th. Camus dir.), Univ. Poitiers, 3 vol.

Puaud Fl. 1992 : Le château de Montreuil-Bonnin [Vienne], mém. maîtr. d'histoire de l'art (M.-Th. Camus dir.), Univ. Poitiers.

Puisais E. 1991 : "Le château de Touffou, Bonnes (Poitou-Charentes)", Cah Pays chauvinois, $n^{\circ} 7,4-5$.

Puissant Fr. 2004 : Vivonne [Vienne]. Origines, topographie et morphologie d'une petite ville du Haut-Poitou (de l'Antiquité au XVIe siècle), mém. maîtr. d'archéologie (L. Bourgeois dir.), Univ. Poitiers, 2 vol.

Ramirez A. 2002 : Le château de Gençay [Vienne], petit mobilier et projet d'exposition, mém. maîtr. d'archéologie (L. Bourgeois dir.), Univ. Poitiers.

Rapin Th. 2000 : "Les grands chantiers du duc de Berry" Bull. Soc. Antiq. Ouest 5e s., t. XIV (paru en 2002), 318.

Raynaud C. 2002 : “ Guy de Dampmartin et la genèse du gothique flamboyant en France ", Cah. archéo., 50, 185-200 [sur le palais de Poitiers].

Reitz S. 1993 : Le château de Tennessus [Deux-Sèvres], mém. maîtr. d'histoire de l'art (M.-Th. Camus dir.), Univ. Poitiers.

Rémy Chr. 2003 : "La vie de château", Actualité Poitou-Charentes, n 61, juillet-septembre 2003, 12-13.

Renoux A. 1994 : Palais médiévaux (France, Belgique). 25 ans d'archéologie, Paris.

Richard Ch. 1990 : "Sites médiévaux et photographie aérienne dans le sud du département de la Vienne : premiers résultats ", Sites défensifs et sites fortifiés au Moyen Age entre Loire et Pyrénées, actes du premier colloque d'Aquitania (1987), Bordeaux, 177-182 (Supplément 4 d'Aquitania).
Rodriguez-Pamias C. 1997 : La céramique du château de Parthenay [DeuxSèvres], mém. maîtr. d'histoire (M. Aurell dir.), Univ. Poitiers.

Sailhan P. 1988 : "Souterrains et salles souterraines à Chauvigny [Vienne] ", Le Pays chauvinois, $\mathrm{n}^{\circ} 26,57-74$.

Sailhan P. 1990 : "Contreforts et tourelles pleines dans les fortifications de Chauvigny ", Le Pays chauvinois, $\mathrm{n}^{\circ} 20-31$.

Sailhan, P. 1995: "Le Château baronnial ou Château des Evêques [à Chauvigny] ", Bull. Soc. amis Pays lochois, n 11, 73-82.

Sailhan P. 1997 : «Le château baronnial ou des évêques de Poitiers à Chauvigny (Vienne)", Cah. Pays chauvinois, 16.

Salamagne A. 1988 : " Pour une approche typologique de l'architecture militaire : l'exemple de la famille monumentale des tours-portes de plan curviligne", Archéo. méd., t. 18, 179-213 [Bressuire, Le Coudray-Salbart].

Senseby Ch. 1995 : Peuplement et société aux XIe et XIIe s. en Touraine méridionale d'après le cartulaire de Noyers, thèse d'histoire nouveau régime, Univ. Paris I-Sorbonne, 2 vol.

Serdon V. 2003 : Etude archéologique de l'armement de trait au Moyen Age (XIe$X V e$ s.), thèse de doctorat nouveau régime (F. Piponnier dir.), Univ. Lyon II, 4 vol. (sous presse).

Servant S. 1995 : Les restaurations du XIXe s. au château de Ternay [Vienne], mém. maîtr. d'histoire de l'art (M.-Th. Camus dir.), Univ. Poitiers.

Servant S. 1996 : Les tours maîtresses du Poitou aux XIe et XIIe s., mém. DEA de civilisation médiévale (M.-Th. Camus dir.), Univ. Poitiers.

Triolet J. et L. 1995 : Les souterrains : le monde des souterrains-refuges en France, Paris, Errance.

Triolet J. et L. 1998 : “ Le souterrain aménagé du château du Cluzeau à LathusSaint-Rémy [Vienne] ". Bull. Soc. Antiq. Ouest, $5^{\mathrm{e}}$ s., t. XII, 59-72.

Uge M.-C. 1994 : "Quelques pavages médiévaux d'Aunis et de Saintonge ", Bull. Soc. archéol. hist. Charente-Maritime, t. 21, 66-81 [châteaux de Merpins, la Folatière et Surgères].

Verdon A. 1994 : “Les tours du Châtelet et d'Harcourt à Parthenay [DeuxSèvres]. Résultats des fouilles, histoire, étude et analyses comparatives ". Bull. Soc. hist. scient. Deux-Sèvres, $3^{\mathrm{e}}$ s., t. II, 327-398.

Vouhé G. 1997 : Le château de Thouars [Deux-Sèvres], mém. maîtr. d'histoire de l'art (V. Meyer dir.), Univ. Poitiers.

Warmoes I. 2000 : "Les fortifications Plantegenêt de La Rochelle", Les fortifications dans les domaines Plantagenêt (XIIe-XIVe s.) (M.-P. Baudry dir.), Poitiers, CESCM, 119-122 et pl. XXIV. 